PROCEEDINGS OF THE

AMERICAN MATHEMATICAL SOCIETY

Volume 34, Number 2, August 1972

\title{
SOME THEOREMS ON FREDHOLM MAPS
}

\author{
A. J. TROMBA
}

Abstract. Let $f: M \rightarrow N$ be a $C^{1}$ proper Fredholm map of index $n$ of $C^{r}$ Banach manifolds. Then under certain hypotheses one may approximate $f$ by a $C^{r}$ proper Fredholm map $g$ of index $n$ with $g$ $C^{1}$ properly homotopic to $f$.

The rank theorem for Fredholm maps is stated and it is then used to obtain the invariance of domain for this class of maps and also a nonlinear version of the Fredholm alternative theorem.

In recent years nonlinear Fredholm maps introduced by Smale [9] have been studied extensively by several authors [1], [2], [5], [6] and applications have been given to nonlinear elliptic boundary value problems [3]. One of the basic tools in the differential theory of such maps is that of approximating a $C^{1}$-Fredholm by one of class $C^{r}, r>1$. In the first section below we show that in certain cases we may approximate a $C^{1}$ proper Fredholm map $f$ by a $C^{r}$ proper Fredholm map $g$ of the same index with $g$ $C^{1}$ properly homotopic to $f$.

In $\S \mathrm{B}$ we state the rank theorem for Fredholm maps and use it to obtain a nonlinear version of the Fredholm alternative theorem and an invariance of domain theorem.

The author is indebted to D. K. Elworthy whose ideas inspired this paper.

A. We begin with a few definitions and basic results. Let $E$ and $F$ be Banach spaces. A linear map $T \in L(E, F)$ is Fredholm if

(i) $\operatorname{dim} \operatorname{Ker} T<\infty$,

(ii) $\operatorname{dim}$ Coker $T<\infty$.

If $M$ and $N$ are Banach manifolds, a $C^{1}$ map $f: M \rightarrow N$ is said to be Fredholm if $d f_{p}: T M_{p} \rightarrow T N_{f(p)}$ is linear Fredholm for each $p \in M$. If $M$ is connected we may define the index of $f$ to be

$$
\operatorname{ind}(f)=\operatorname{dim} \operatorname{Ker} d f_{p}-\operatorname{dim} \text { Coker } d f_{p} .
$$

This does not depend on the choice of $p$.

A point $y \in N$ is said to be a regular value of $f$ if whenever $x \in f^{-1}(y)$, $d f_{x}: T M_{x} \rightarrow T N_{y}$ is surjective. If $y$ is not a regular value it is a critical value.

Received by the editors February 16, 1971.

AMS 1970 subject classifications. Primary 58B15; Secondary 45N05.

(c) American Mathematical Society 1972 
The following generalization of the Sard theorem due to Smale is the cornerstone of the modern differential theory of Fredholm maps.

TheOREM 1 (SARD-SMALE). Let $M$ be a second countable Banach manifold with $f: M \rightarrow N$ a $C^{3}$ Fredholm map of index $n$. Then if $q>\max (n, 0)$ the regular values are of second category in $N$ (or the critical values are of first category). If $f$ is proper (i.e., $K$ compact $\Rightarrow f^{-1}(K)$ compact) then the regular values are open and dense.

Let $M$ be a $C^{1}$ Finsler manifold [7]. Then $M$ admits a metric $\rho$ (the Finsler metric). A set $X \subset M$ is bounded if, with respect to the metric $\rho$, $\operatorname{diam}(X)=\sup _{x, y \in X} \rho(x, y)<\infty$.

Suppose $N$ is another Finsler manifold with a Finsler metric $\rho^{\prime}$. Given maps $f: M \rightarrow N$ and $\delta: M \rightarrow R_{+}$, a map $g: M \rightarrow N$ is said to be a $\delta$-approximation to $f$ if $\rho^{\prime}(f(x), g(x)) \leqq \delta(x)$ for all $x \in M$.

The topology on $C(M, N)$, the continuous maps from $M$ to $N$ generated by neighborhoods of the form

$$
W(f, \delta)=\left\{g \in C(M, N) \mid \rho^{\prime}(f(x), g(x))<\delta(x)\right\}
$$

where $\delta: M \rightarrow R_{+}$is continuous, is called the $C^{0}$-fine topology on $C(M, N)$. It is not difficult to show that $C^{0}$ approximation does not depend on the metric $\rho^{\prime}$.

Now again let $M, N$ be Finsler manifolds with metrics $\rho, \rho^{\prime}$. A map $h: M \rightarrow N$ is called compact if the image of $\rho$-bounded sets is relatively compact in $N$.

Definition. Suppose $f_{0}: M_{0} \rightarrow E_{0}$ is a $C^{1}$ Fredholm map of index $n$ of the $C^{r}$ Finsler manifold $M_{0}$, modelled on $F_{0}$ with metric $\rho_{0}$, to the Banach space $E_{0}$. A smoothing space of class $C^{r}$ for the triple $\left(f_{0}, M_{0}, E_{0}\right)$ is a triple $\left(f_{1}, M_{1}, E_{1}\right)$ where $M_{1}$ is a $C^{r}$ Finsler manifold modelled on $F_{1}$ admitting $C^{r}$ partitions of unity with metric $\rho_{1}, \rho_{1} \leqq k \rho_{0}, k$ some positive constant. In addition we assume $M_{0} \subset M_{1}, E_{0} \subset E_{1}, F_{0} \subset F_{1}$ are dense inclusions with the inclusion map $i: M_{0} \rightarrow M_{1}$ compact. We assume also the $f_{1}$ is Fredholm of index $n$ making the diagram

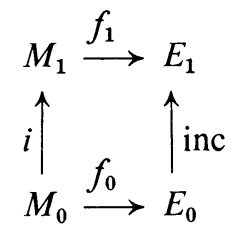

commutative. Lastly we suppose that there is a differential structure on $M_{1}$ uniform with respect to $M_{0}$; that is a maximal atlas of charts on $M_{1},\left(V_{\beta}, \psi_{\beta}\right)_{\beta \in B}$ with $\psi_{\beta} \mid V_{\beta} \cap M_{0}$ a $C^{1}$ chart for $M_{0}$. 
The smoothing space is second countable if $M_{1}$ is. To see how smoothing spaces arise in the study of nonlinear elliptic boundary value problems see [3].

The proof of the following theorem is a minor modification of the argument in [3].

THEOREM 2. Let $f: M_{0} \rightarrow E_{0}$ be Fredholm of index $n$. If there exists a $C^{\mathbf{1}}$ smoothing space for the triple $\left(f_{0}, M_{0}, E_{0}\right)$ then $f_{0}$ is a proper map when restricted to bounded subsets of $M_{0}$.

The proof of this theorem involves the notion of a $C$-structure on a Banach manifold which we now define. Let $\mathrm{GL}_{c}(E) \subset \mathrm{GL}(E)$ be the subgroup of the general linear group of $E$ consisting of elements of the form $I+K, K$ completely continuous. A $C$-structure on a manifold $M$ is a maximal atlas of charts $\left(U_{\alpha}, \varphi_{\alpha}\right)_{\alpha \in A}$ covering $M$ so that when defined $D\left(\varphi_{\alpha} \circ \varphi_{\beta}^{-1}\right) \varphi_{\beta}(x) \in \mathrm{GL}_{c}(E)$.

A linear operator $T \in L\left(E \times R^{n}, E\right)$ is a $C\left(\pi_{n}\right)$ linear map if $T=\pi_{n}+K$, where $\pi_{n}: E \times R^{n} \rightarrow E$ is the natural projection on the first factor and $K$ is completely continuous (here we take $\pi_{0}=\mathrm{id}_{E}$ ). Suppose $M$ is modelled on $E \times R^{n}$ as a $C$-structure $M_{c}$. A Fredholm map $f$ of index $n$ is a $C\left(\pi_{n}\right)$ map if, with respect to charts $(\varphi, V) \in M_{c}, D\left(f \circ \varphi^{-1}\right)_{x}$ is $C\left(\pi_{n}\right)$.

The following basic result concerning $C\left(\pi_{n}\right)$ maps is proved in [2].

THEOREM 3. Let $M$ be modelled on $F, f: M \rightarrow E$ Fredholm of index $n$. Then there exists a unique $C$-structure $\{M, f\}_{c}$ on $M$ modelled on $E \times R^{n}$ with respect to which $f$ is a $C\left(\pi_{n}\right)$ map.

REMARK. If $\left(f_{1}, M_{1}, E_{1}\right)$ is a smoothing space for $\left(f_{0}, M_{0}, E_{0}\right)$ the induced $C$-structure on $M_{1}$ can be made uniform with respect to $M_{0}$.

Before getting to the main result we will need a sequence of three lemmas.

Lemma 1. Let $U \subset E \times R^{n}$ be an open subset where $E$ admits $C^{r}, r>1$, partitions of unity. Suppose $f: U \rightarrow E$ is $a C^{1} C\left(\pi_{n}\right)$ map. Then given any $\delta>0$ there exists a $C^{r} C\left(\pi_{n}\right)$ map $g$ with $\|g(x)-f(x)\|<\delta$. Moreover, if $E_{0} \subset E$ is dense, $g$ can be chosen so that $g(x)-\pi_{n}(x) \in E_{0}$.

Proof. Let $V_{y}(\delta / 2)$ be a covering of $E$ by balls of radius $\delta / 2$ about the point $y$ (we may choose $y \in E_{0}$ ), and let $\left\{W_{y}\right\}_{y \in E}$ be an open cover of $U$ defined by $W_{y}=\hat{f}^{-1}\left(V_{y}\right)$ where $\hat{f}(x)=f(x)-\pi_{n}(x)$.

Let $\left\{\hat{W}_{j}\right\}_{j \in J}$ be a locally finite open refinement of $\left\{W_{y}\right\}_{y \in F}$ covering $U$ with $\left\{\varphi_{j}\right\}$ a $C^{r}$ partition of unity subordinate to the covering $\left\{\hat{W}_{j}\right\}$. Choose $V_{y_{j}}$ so that $\hat{f}\left(\hat{W}_{j}\right) \subset V_{y_{j}}$. Define

Then

$$
\hat{g}(x)=\sum \varphi_{j}(x) y_{j}, \quad g(x)=\pi_{n}(x)+\hat{g}(x) .
$$

$$
\|f(x)-g(x)\|=\|\hat{f}(x)-\hat{g}(x)\|=\left\|\sum \varphi_{j}(x)\left\{\hat{f}(x)-y_{j}\right\}\right\| .
$$


If $\left\|\hat{f}(x)-y_{j}\right\|>\delta / 2$ then $\varphi_{j}(x)=0$. Thus $\|\hat{f}(x)-\hat{g}(x)\|<\delta$. Moreover since $x \mapsto \sum \varphi_{j}$ is locally finite dimensional, $g$ is a $C^{r} C\left(\pi_{n}\right)$ map and satisfies the requirements of the lemma.

Lemma 2. Suppose $\delta: U \rightarrow R_{+}$is continuous. Then the above lemma is true for nonconstant $\delta$.

Proof. For each $x$, let

$$
W_{x}=\{z \in U|| \delta(z)-\delta(x) \mid<\delta(x) / 2\} .
$$

Let $\left\{W_{j}\right\}_{j \in J}$ be a locally finite open refinement of $\left\{W_{x}\right\}_{x \in U}$ covering $U$, and let $x_{j}$ be chosen so that $W_{j} \subset W_{x_{j}}$. Let $f_{j}: W_{j} \rightarrow F$ be the restriction of $f$ to $W_{j}$ and $\left\{\mu_{j}\right\}_{j \in J}$ be a $C^{r}$ partition of unity subordinate to $W_{j}$. By the last lemma there is a $C^{r} C\left(\pi_{n}\right)$ map with $\left\|g_{i}(x)-f_{i}(x)\right\|<\delta\left(x_{i}\right) / 2$. Then $g(x)=$ $\sum \mu_{j}(x) g_{j}(x)$ will be a $C\left(\pi_{n}\right)$ map of $U$ onto $E$ and

$$
\|g(x)-f(x)\| \leqq \sum \varphi_{j}(x)\left\|g_{j}(x)-f(x)\right\| \leqq \sum \varphi_{j}(x)\left(\delta\left(x_{j}\right) / 2\right) .
$$

If $x \in W_{j}, \delta\left(x_{j}\right) / 2<\delta(x)$ which implies that $\|g(x)-f(x)\|<\delta(x)$.

LEMmA 3. Let $W, V, U$ be open sets in $E \times R^{n}$ with $\bar{W} \subset V \subset \bar{V} \subset U$ and $f: U \rightarrow E$ a $C^{1} C\left(\pi_{n}\right)$ map and $\delta: U \rightarrow R_{+}$continuous. Then there exists a $C^{r}$ $C\left(\pi_{n}\right)$ map $g: U \rightarrow E$ with $g \mid W$ a $C^{r} C\left(\pi_{n}\right)$ map $g|(U-\bar{V})=f|(U-\bar{V})$ and $\|f(x)-g(x)\|<\delta(x)$. Moreover there is a $C^{1}$ homotopy $f_{t}$ of $f$ so that $f_{0}=f$, $f_{1}=g$ with $f_{t}|(U-\bar{V})=f|(U-V)$ and $\left\|f_{t}(x)-f(x)\right\|<\delta(x)$ for all $t \in[0,1]$.

Proof. Consider $\hat{f}=f \mid V$. By Lemma 2 there is a $C^{r} \delta$-approximation $\hat{g}: V \rightarrow F$ to $\hat{f}$ which is a $C\left(\pi_{n}\right)$ map. Let $\left\{\mu_{i}\right\}_{i=1,2}$ be a partition of unity subordinate to the covering $V, U-\bar{W}$ of $U$ with support of $\mu$, contained in $V$. Define $\hat{g}(x)=\mu_{1}(x) \hat{g}(x)+\mu_{2}(x) f(x)$ and $f_{t}=(1-t) g+t f$.

REMARK. A slight modification of Lemma 3 shows that if $f$ is a $C^{r}$ $C\left(\pi_{n}\right)$ map on a neighborhood of a closed set $A \subset U$ then we can choose $g$ so that $g|A=f| A$, and the same for $f_{t}$.

THEOREM 4. Let $f_{0}: M_{0} \rightarrow E_{0}$ be a Fredholm map of index $n$ with $C^{r}$ smoothing space $\left(f_{1}, M_{1}, E_{1}\right)$. Then given $\delta: M_{1} \rightarrow R_{+}$there exists a $C^{r}$ Fredholm map $g_{0}: M_{0} \rightarrow E_{0}$ of index $n$ which is a $\delta$-approximation to $f_{0}$ and admits a smoothing space $\left(g_{1}, M_{1}, E_{1}\right)$. Moreover there exists a homotopy $F_{0}: M_{0} \times I \rightarrow E_{0}$ between $f$ and $g$ which has a $C^{r}$ smoothing space $\left\{F_{1}, M_{1} \times I\right.$, $\left.E_{1}\right\}, F_{1}^{t}$ a homotopy between $g_{1}$ and $f_{1}$ which is a $\delta$-approximation to ffor each $t \in[0,1]$.

Proof. We first assume that $M_{1}$ is second countable, thus all index sets will be countable. By Theorem $3, M_{1}$ admits a $C$-structure $\left\{M_{1}, f_{1}\right\}_{c}$ with respect to which $f_{1}$ is a $C\left(\pi_{n}\right)$ map and the $C$-structure is uniform with respect to $M_{0}$. 
Let $U_{i}$ be an open cover of $M_{1}$ with $U_{i}$ bounded and $\left(U_{\alpha}, \varphi_{\alpha}\right)$ a coordinate system in the $C$-structure $\left\{M_{1}, f_{1}\right\}_{c}$. Let $\left\{G_{\alpha}\right\},\left\{V_{\alpha}\right\}$ be locally finite open coverings of $M_{1}$ with $\bar{G}_{\alpha} \subset V_{\alpha}, \bar{V}_{\alpha} \subset U_{\alpha}$, $\operatorname{dist}\left(\bar{G}_{\alpha}, V_{\alpha}^{c}\right)>0$. Let $\eta<\delta$ and $g_{0}=f$. Lemma 3 gives us a map $g_{1}^{1}: M_{1} \rightarrow E_{1}$ which is a $C\left(\pi_{n}\right)$ map on $W_{1}$ where $\bar{G}_{1} \subset W_{1}, \bar{W}_{1} \subset V_{1}$, $\operatorname{dist}\left[\bar{G}_{1}, W_{1}^{c}\right]>0$, and $g_{1}^{1}$ is equal to $f$ on the complement of $V_{1}$. Moreover we get that $g_{1}^{1}$ is $C^{1}$ homotopic to $f$ by just a linear homotopy, and it is clear that $g_{1}^{1}$ restricts to $M_{0}$ to give an approximation $g_{0}^{1}$ to $f_{0}$.

Suppose we have defined a $C\left(\pi_{n}\right)$ map $g_{1}^{s-1}: M_{1} \rightarrow E_{1}$ which restricts to a $C\left(\pi_{n}\right)$ map $g_{0}^{s-1}: M_{0} \rightarrow E_{0}$ where $g_{1}^{s-1}$ is $C^{r}$ on a neighborhood $W_{s-1}$ of $\bigcup_{k=1}^{s-1} \bar{G}_{k}$ which is a $\left(1-1 / 2^{s-1}\right) \eta$ approximation to $f$. Let $A_{s}=\bigcup_{k=1}^{s} \bar{G}_{k}$ and take open neighborhoods $H$ of $A_{s-1}$ and $B$ of $\bar{G}_{s}$ with $\bar{H} \subset W_{s-1}$ and $\bar{B} \subset V_{s}$. By the remark after Lemma 3 there is a map $\bar{g}_{1}^{s}: U_{s} \rightarrow E_{1}$ which is a $C^{r} C\left(\pi_{n}\right)$ map on $B$ and equal to $g_{1}^{s-1}$ outside $V_{s}$ and on $V_{s} \cap \bar{H}$ and also on $\eta / 2^{s}$ approximation to $g_{1}^{s-1}$. This extends over $M_{1}$ to give a map $g_{1}^{s}: M_{1} \rightarrow E_{1}$ which is $C^{r} C\left(\pi_{n}\right)$ in a neighborhood of $A_{s}$ restricts to $g_{1}^{s-1}$ on $A_{s-1}$, is a $\left(1-1 / 2^{s}\right) \eta$ approximation to $f$ and is homotopic to $g_{1}^{s-1}$ by a homotopy which is constant on $A_{s-1}$. Moreover all $C^{r}$ maps restrict naturally by construction to $C^{r}$ maps from $M_{0}$ to $E_{0}$.

Thus we can define the map $g_{1}: M_{1} \rightarrow E_{1}$ and hence $g_{0}: M_{0} \rightarrow E_{0}$ inductively. If $M_{1}$ is not second countable we obtain the result using the same method but with refinements of the type considered in Lemma 2.4 of [8].

Before proving the main theorem of this section we need a definition.

Definition. Let $(M, \rho),\left(N, \rho^{\prime}\right)$ be Finsler manifolds with metrics $\rho$ and $\rho^{\prime}$ respectively. A map $f: M \rightarrow N$ is $B$-proper if:

(i) if $X \subset M$ is bounded, $f \mid X$ is proper;

(ii) if $Y \subset N$ is bounded then $f^{-1}(Y)$ is bounded in $X$.

It is clear that $B$-proper maps are proper. In addition in applications to nonlinear elliptic boundary value problems it is the $B$-properness that one usually verifies.

THEOREM 5. Let $f_{0}: M_{0} \rightarrow E_{0}$ be a $C^{1}$ B-proper Fredholm map of index $n$, with $C^{r}$ smoothing space $\left(f_{1}, M_{1}, E_{1}\right)$. Then given $\delta: M_{1} \rightarrow R_{+}$there is a $C^{r} \delta$-approximation $g_{0}$ to $f_{0}$ which is B-proper Fredholm of index $n$. Moreover there is a B-proper map $F_{0}: M_{0} \times I \rightarrow E_{0}$ so that for each $t \in I, F_{0}^{t}$ is a Fredholm map of index $n$, and a $\delta$-approximation to $f_{0}$.

Proof. Let $\eta<\delta<1$. The last theorem gives an $\eta$-approximation $g$ to $f$ with $C^{r}$ smoothing space $\left(g_{1}, M_{1}, E_{1}\right)$, and also a homotopy $F_{0}^{t}$ of $f_{0}$ and $g_{0}$. By Theorem $2, g_{0}$ is proper on bounded sets. Also $\left\{x \mid\left\|g_{0}(x)\right\| \leqq C\right\} \subseteq$ $\left\{x \mid\left\|f_{0}(x)\right\| \leqq C+1\right\}$ which shows that $g_{0}$ is $B$-proper if $f_{0}$ is. The $B$-properness of $F_{0}$ follows similarly. 
Remark. These results easily extend to the case where $E_{0}$ is replaced by a Finsler manifold $N_{0}$ with a fixed $C$-structure.

B. Let $f: M \rightarrow N$ be a proper $\mathscr{F}_{0}$ (Fredholm of index zero) map between two Banach manifolds. In [9] Smale showed that the cardinality of $f^{-1}(y) \bmod 2$, for $y$ a regular value, was independent of the point $y$. The mod 2 degree of $f$, denoted by $\gamma(f)$, has the property that if $\gamma(f) \neq 0$ then $f$ must be surjective. In this section we will use this fact and an infinite dimensional version of the rank theorem to obtain a nonlinear extension of one of the standard Fredholm alternative theorems (see Widom [10]).

Theorem 1 (RANK TheOREM For Fredholm mappings). Let $b \in A \subset E$, $A$ open, $f: A \rightarrow F$, a Fredholm mapping of index $p$ with $\operatorname{dim} \operatorname{Ker} D f_{x}=s$ for $x \in A$. Then there exists a Banach space $G$, neighborhoods $U$ of $b$ and $V \supset f(U)$ of $f(b)$ and diffeomorphisms $\varphi: B_{s} \rightarrow U, \psi: V \rightarrow B_{s-p}$, where $B_{k}$ is the open unit ball in $G \times E^{k}$, such that

$$
\psi \circ f \circ \varphi=\mathrm{id}_{G} \times 0 .
$$

The proof is a modified version of the one for finite dimensions.

Lemma. Suppose $\xi: \Gamma \rightarrow \mathscr{F}(E, F)$ is a continuous map of a topological space $\Gamma$ into Fredholm operators from $E$ to $F$. Then there exists a point $b \in \Gamma$ and a neighborhood $U$ of $b$ such that for $x \in U$, $\operatorname{dim} \operatorname{Ker} \xi(x)$ is constant.

Proof. Let $X_{s}$ be the closed subset of $X$ defined by

$$
X_{s}=\{x \in \Gamma \mid \operatorname{dim} \operatorname{Ker} \xi(x) \geqq s\} .
$$

Let $s_{0}$ be the smallest $s$ so that $\sim X_{s} \neq \varnothing$ for $s>s_{0}$ or equivalently $X_{s} \neq$ $X_{s_{0}}=X$, for $s>s_{0}$. Let $b \in X$ be chosen so that $\operatorname{dim} \operatorname{Ker} \xi(b)=s_{0}$, and $U$ be an open neighborhood of $b$ with the property that $\operatorname{dim} \operatorname{Ker} \xi(x) \leqq$ $\operatorname{dim} \operatorname{Ker} \xi(b)$ for $x \in U$.

Theorem 2. Suppose $f: M \rightarrow N$ is a $C^{1}$ injective Fredholm map between two Banach manifolds. Then the set $S(f)=\left\{x \mid D f_{x}\right.$ not injective $\}$ of singularities of $f$ is nowhere dense in $M$.

Proof. Clearly $S(f)$ is closed. Suppose $S(f)$ contains an open set, then we may work locally and assume $M$ is open in $E, N$ open in $F$ and $S(f)$ contains an open ball $B$ about one of its points, so $B \subset S(f)$. Define $\xi: B \rightarrow \phi(E, F)$ by $\xi(x)=D f_{x}$. From the lemma it follows that there is an open set $A \subset B$ on which $\operatorname{dim} \operatorname{Ker} \xi(x)=s>0$.

Applying Theorem 1 we obtain a representative of $f$ which contradicts the fact that $f$ is an injection. 
THEOREM 3. Let $f: M \rightarrow N$ be a proper $\mathscr{F}_{0}$ map between two Banach manifolds with $N$ connected. If $f$ is injective then it is surjective.

Proof. By Theorem 2 there is a point $x_{0}$ with $D f_{x_{0}}$ a linear injection. Thus $y=f\left(x_{0}\right)$ is a regular value, $f^{-1}(y)=x_{0}$, and $\gamma(f)=1$.

The above proposition is clearly false if we take maps which are not proper. For example the map which takes the plane homeomorphically onto the two-sphere minus the north pole is Fredholm of index zero, but not onto the sphere, however the map is open. This is summarized in the following.

THEOREM 4 (INVARIANCE OF DOMAIN). Let $f: M \rightarrow N$ be a locally injective $\mathscr{F}_{0}$ map. Then $f$ is an open map.

Proof. Let $y=f(x)$. We must show there is a neighborhood of $x$ which is mapped to a neighborhood of $y$. Choose a connected open neighborhood $U$ of $x$ in $M$ such that $f \mid \bar{U}$ is proper and injective. Then $y \notin f(\partial U)$. Let $V$ be the connected component of $N-f(\partial U)$ containing $y$. Since $f \mid \bar{U}$ is proper, $f(\partial U)$ is closed and so $V$ is open in $N$. Moreover $f U \subset V$ by connectedness. Finally, since $\bar{U} \cap f^{-1} V=U$, the $\operatorname{map} f \mid U: U \rightarrow V$ is proper. By Theorem 3 it is therefore onto. Hence $f U(=V)$ is open.

TheOrem 5 (nONLINEAR Fredholm ALternative). Let $f: M \rightarrow N$ be $a$ locally injective proper $\mathscr{F}_{0}$ map. If $N$ is connected then $f$ maps $M$ onto $N$ and is a covering map.

Proof. By Theorem $4, f(M)$ is open in $N$. Since $f$ is proper it is also closed and thus all of $N$. From the fact that $f$ is an open map it follows that it is a local homeomorphism and then the properness of $f$ implies that $N$ is locally evenly covered by $f$.

THEOREM 6. If $f: M \rightarrow N$ is as in Theorem 5 with $M$ connected and $N$ simply connected then $f$ is a homeomorphism.

Proof. Follows from a standard covering space argument.

The above theorems can be proven by passing to local coordinates and using results already known for compact fields, e.g. Granas [4], however the results illustrate the natural beauty and simplicity of degree theory arguments. We conclude this section with a generalization of a lemma of M. Hirsch to Banach manifolds. Using it one can, in special cases, prove the classical Schauder fixed point theorem. The proof of the following is essentially the same as in finite dimensions.

THEOREM 7. Let $X$ be a Banach manifold with boundary $\partial X$ (also a smooth Banach manifold). Then there exists no proper $\mathscr{F}_{0}$ map $f: X \rightarrow X$ with $f(X) \subset \partial X$ and $f \mid \partial X=\mathrm{id}_{\partial X}$; i.e., $\partial X$ is not a proper $\mathscr{F}_{0}$ retract of $X$. 


\section{BIBLIOGRAPHY}

1. J. Callahan, Intersection theory for infinite dimensional manifolds, Thesis, New York University, 1967.

2. D. Elworthy and A. Tromba, Differential stkuctures and Fredholm maps on Banach manifolds, Proc. Sympos. Pure Math., vol. 15, Amer. Math. Soc., Providence, R.I., 1970, pp. 45-93.

3. - Degree theory on Banach manifolds, Proc. Sympos. Pure Math., vol. 18, Amer. Math. Soc., Providence, R.I., 1970.

4. A. Granas, The theory of compact vector fields and some of its applications to the topology of functional spaces. I, Rozprawy Mat. 30 (1962), 93 pp. MR 26 \#6743.

5. K. K. Mukherjea, Coincidence theorems for infinite dimensional manifolds, Bull. Amer. Math. Soc. 74 (1968), 493-496. MR 36 \#5965.

6. U. Koschocke, Infinite dimensional $K$-theory and characteristic classes of Fredholm bundle maps, Proc. Sympos. Pure Math., vol. 15, Amer. Math. Soc., Providence, R.I. 1970.

7. R. Palais, Lusternik-Schirlman theory on Banach manifolds, Topology 5 (1966), 115-132. MR 41 \#4584.

8. - — Homotopy theory of infinite dimensional manifolds, Notes, Brandeis University, Waltham, Mass., 1967.

9. S. Smale, An infinite dimensional version of Sard's theorem, Amer. J. Math. 87 (1965), 861-866.

10. H. Widom, Integral equations, Van Nostrand, Princeton, N.J., 1968.

Department of Mathematics, University of California, Santa Cruz, CaliFORNIA 95060 